\title{
Visualizing the demand for various resources as a function of the master surgery schedule: A case study
}

\author{
Jeroen Beliën \\ Erik Demeulemeester \\ Brecht Cardoen \\ Katholieke Universiteit Leuven \\ Faculty of Economics and Applied Economics \\ Department DSIM: Decision Sciences 8 Information Management \\ Research Center for Operations Management \\ Naamsestraat 69, B-3000 Leuven, Belgium \\ email: jeroen.belien@econ.kuleuven.be \\ email: erik.demeulemeester@econ.kuleuven.be \\ email: brecht.cardoen@econ.kuleuven.be \\ phone: 0032 (0)16326972
}




\begin{abstract}
This paper presents a software system that visualizes the impact of the master surgery schedule on the demand for various resources throughout the rest of the hospital. The master surgery schedule can be seen as the engine that drives the hospital. Therefore, it is very important for decision makers to have a clear image on how the demand for resources is linked to the surgery schedule. The software presented in this paper enables schedulers to instantaneously view the impact of, e.g., an exchange of two block assignments in the master surgery schedule on the expected resource consumption pattern. A case study entailing a large Belgian surgery unit illustrates how the software can be used to assist in building better surgery schedules.
\end{abstract}

Keywords: Operating room scheduling, visualization, resource management, case study

\title{
1 Introduction
}

The operating room can be seen as the engine that drives the hospital (Litvak and Long, 2000). Indeed, what happens inside the operating room dramatically influences the demand for resources throughout the rest of the hospital. For instance, after surgery, a patient often occupies a bed and requires nursing services for recovery. Certain types of surgery entail preceding tests like blood analysis or post-surgery treatments that have to be carried out by correctly skilled staff. Consequently, the demand patterns for these resources are highly dependent on the operating room schedule. The software system described in this paper visualizes the impact of the master surgery schedule on the demand for all kinds of resources like beds, staff (nurses, anaesthetists, etc.), specialized equipment, radiology and so on.

Hamilton and Breslawski (1994) argue that the factors considered by operating room administrators to be critical to operating room scheduling are dependent on the nature of the scheduling system. The results of their large scale survey indicated that in block systems, which is the system used in this case study, the number of operating rooms, the equipment limitations, the block times assigned and the hospital scheduling policy 
are considered to be important criteria. In first come, first served systems the number of operating rooms, the estimated room set up duration, the estimated case duration and the equipment restrictions are considered to be essential.

The management of resources is often considered a crucial issue in operating room scheduling. Ozkarahan (1995) proposes an expert hospital decision support system for resource scheduling that combines mathematical programming, knowledge base, and database technologies. Five years later, the same author (Ozkarahan, 2000) describes a goal programming model that can produce schedules that best serve the needs of the hospital, i.e., by minimizing idle time and overtime, and increasing satisfaction of surgeons, patients and staff. The approach involves sorting the requests for a particular day on the basis of block restrictions, room utilization, surgeon preferences and intensive care capabilities. Certain types of operating room related resources have gained much attention in the literature so far. The relation between bed occupancy and the surgery schedule has been subject to many studies (e.g., Dumas, 1984 and 1985; Harris, 1985; Wright, 1987; Clerkin et al., 1995; Gorunescu et al., 2002; McManus et al., 2004; Santibanez et al., 2005). Also, operating room staffing problems have been studied in many papers. Dexter and Traub (2000), for instance, determine staffing requirements for a second shift of anaesthetists by graphical analysis of data from operating room information systems. Dexter et al. (2000) use computer simulation to investigate the effects of scheduling strategies on operating room labor costs. Griffiths et al. (2005) model the requirement for supplementary nurses in an intensive care unit.

The surgery scheduling process for elective cases is often seen as a three stage process (Blake and Donald, 2002). The model described in this paper is situated in the second stage and as such distinguishes itself from studies situated in the first or the third stage. The first stage concerns the long term case mix planning. In this stage, it is determined how much operating room time is assigned to the different surgeons (or surgical groups). Case mix planning problems have been studied by amongst others Hughes and Soliman (1985), Rifai and Pecenka (1989), Robbins and Tuntiwongbiboon (1989) and Blake and Carter (2002) and (2003). 
The second stage concerns the development of a master surgery schedule. In the hierarchical framework for hospital production and control by Vissers et al. (2001) this second stage of operating room scheduling can be positioned somewhere between the Resource Planning \& Control level and the Patient Group Planning \& Control level. The master surgery schedule is a cyclic timetable that defines the number and type of operating rooms available, the hours that rooms will be open, and the surgical groups or surgeons who are to be given priority for the operating room time (Blake et al., 2002). Santibanez et al. (2005) present a system-wide optimization model for block scheduling that enables managers to explore trade-offs between operating room availability, booking privileges by surgeons, bed capacity and waitlists for patients. Blake et al. (2002) propose an integer programming model that minimizes the weighted average undersupply of operating room hours, that is allocating to each surgical group a number of operating room hours as close as possible to its target operating room hours (see also Blake and Donald, 2002).

After the development of the master surgery schedule, elective cases can be scheduled. This third stage occurs on a daily base and involves detailed planning of each case. Hans et al. (2005) address the problem of assigning elective surgeries to operating rooms in such a way that not only the utilization of the OR theatre department is optimized, but also the total overtime is minimized. Other interesting work that applies on the third stage has been done by Weiss (1990), Lapierre et al. (1999), Dexter et al. (1999) and (2001), Dexter and Traub (2002), Guinet and Chaabane (2003) and Marcon et al. (2003).

It has been widely accepted that visualization is a simple yet powerful tool for managing complex systems like health care service units. Strum et al. (1997) propose a resource coordination system for surgical services (RCSS) using distributed communications. They present user interfaces that are designed to mimic paper lists and worksheets used by health care providers. These providers enter and maintain patient-specific and site-specific data, which are broadcasted and displayed for all providers. The basic differ- 
ence between RCSS and our system is that RCSS is designed to work online, preventing and solving resource capacity problems by effective communication, while our system works offline and is designed to facilitate the development of better long term cyclic surgery schedules. Carter (2000) describes the successful application of a commercial package, called ORSOS, which is an enterprise-wide surgery scheduling and resource management system. The system autonomically manages all of the hospitals' surgical staff, equipment and inventory using an engine that considers all of the clinical, financial and operational criteria that must be addressed for each surgical event. The difference with our system is that the emphasis lies on the third stage, the detailed elective surgery scheduling, while our system is designed for the second stage.

Simulation packages are often used to analyze and visualize surgical units. Good surveys of simulation approaches in health care clinics can be found in Klein et al. (1993), Jun et al. (1999) and Standridge (1999). Simulation models that focus on the bed occupancy can be found in Dumas (1984) and (1985) and Wright (1987). A specific simulation model for predicting nursing staff requirements has been described by Duraiswamy et al. (1981). Swisher et al. (2001) highlight the graphical visualization features of their object-oriented simulation package for health care clinics. The advantage of simulation, compared to our system, is the capability to analyze stochastic processes and to model more complex discrete-event like relationships. The disadvantage is that building a good simulation model is often very time and cost intensive, which makes it less suitable for quickly analyzing simple what-if scenarios, e.g., for assisting in the development of a new cyclic surgery schedule.

The purpose of the system presented in this paper is threefold. First, schedulers can use it for detecting resource conflicts and constructing workable schedules. Second, the system can greatly assist during the master surgery schedule bargaining process. Visualizing a resource conflict is often far more convincing than hours of discussion with unsatisfied surgeons for not being scheduled by their preferences. Third, the system can be of great value for persuading hospital managers to invest in extra resource capacity. Insufficient resource capacities may not always be visible at first sight. It may, for 
instance, be the case that, although enough resource capacity is available for the individually summed needs for all resources over all surgeons, still no schedule can be found that provides enough capacity of each resource for each surgeon at each time instance.

The remainder of this paper is structured as follows. Section 2 explains the underlying model. Section 3 introduces the surgical unit that is the subject of the case study. Section 4 presents the graphical user interface of the software, providing the reader with a visualization of the surgery schedule and its impact on the resource consumption. Finally, Section 5 draws conclusions and lists some topics for future research.

\section{Underlying model}

Figure 1 contains the underlying model for the visualization software presented in this paper. On top one can see a number of ovals representing the surgeons (or surgical groups). Each surgeon obtains a number of blocks in the schedule. Each block allocation consumes a number of resources represented by the grey ovals. With each resource a consumption pattern can be associated that indicates for each time instance how many units are used. These time instances are relative to the moment of surgery. Time instance " 0 " is during the period of surgery. Time instance "- 1 " indicates one period earlier, e.g., certain types of surgery require preceding tests. Time instance "1" indicates one period later, e.g., the resources needed while the patient is waking up and recovering from surgery. These resource consumption patterns are indicated by the two-row strings at the bottom of Figure 1 . The first row contains the time index $i$, the corresponding cell in the second row gives the required number of units $d_{i}^{k}$ for resource $k$.

In the field of project scheduling, one makes a distinction between renewable and nonrenewable resources (see, e.g., Demeulemeester and Herroelen, 2002). Renewable resources are available on a period-by-period basis, that is the amount is renewable from period to period. Only the total resource use at every time instant is constrained. Typical examples of renewable resources include manpower, equipment, machines, tools and space. On the contrary, nonrenewable resources do not become repeatedly available. 


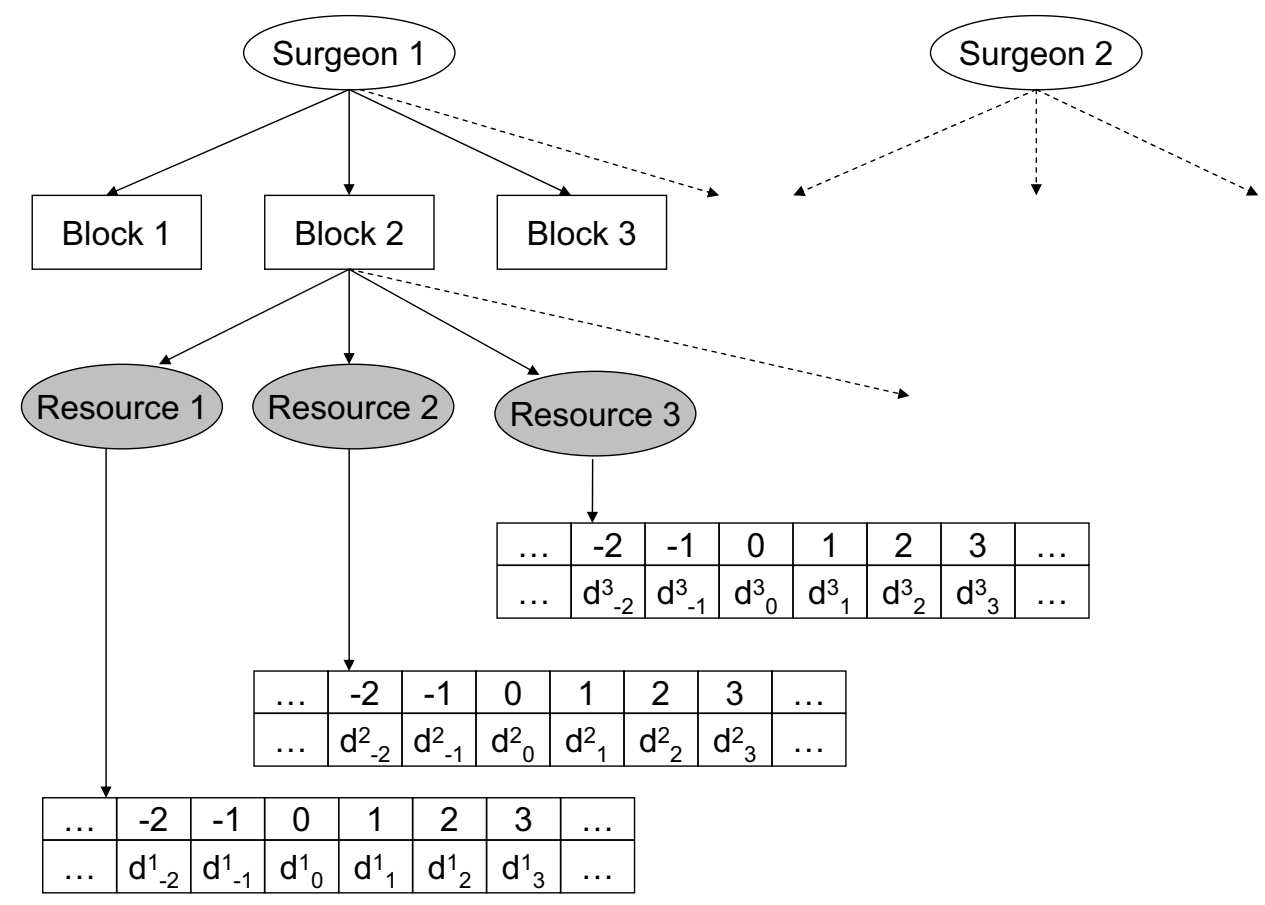

Figure 1: Underlying model

Instead, they have a limited consumption availability for the entire duration that the schedule is employed. Money is perhaps the best example of a nonrenewable resource: the overall budget to span a certain time period (e.g., one year) is frequently predetermined to a fixed amount of money.

Only renewable resources could be modeled in the visualization software presented hereafter. The granularity of the time axis may differ from resource to resource and is not necessarily identical to that of the surgery schedule. As non-renewable resources tend to coincide with case mix decision issues, they are left outside the scope of our visualization software.

Observe that the model does not deal with stochastic data: all resource consumption patterns are assumed to be deterministic. In Beliën and Demeulemeester (2006), 
a theoretical model is proposed that can be seen as a generalization, as well as a particularization, of the model presented in this paper. It can be seen as a generalization, because it also takes uncertainty into account. The model is, however, also more specific than this one, as beds are the only resource taken into consideration. The model starts from stochastic distributions for patient arrivals and a stochastic length of stay (LOS) associated with each type of surgery. The objective is to obtain a leveled bed occupancy distribution and the master surgery schedule is also the instrument to achieve this objective.

\section{Case study}

This case study concerns the day surgery center of the university hospital Gasthuisberg, situated in Leuven, Belgium. As the name suggests, the day surgery center processes only outpatient admissions. To give an idea of the size of this surgical unit, in 2004 12,778 surgical interventions have been performed, making up for more than 15,000 hours of total net operating time.

Gasthuisberg's day surgery operating room complex consists of 8 rooms in which, in total, 27 different surgical entities, divided over 13 surgical and medical disciplines, have been assigned operating room time. Each operating room is open from Monday to Friday from 07.45 am till $4.00 \mathrm{pm}$. No elective surgery takes place during the weekends. Each operating room is allocated for at least half a day to the same surgeon. The current master surgery schedule can be called cyclic since it basically repeats each week with the exception of three block allocations that alter each week between two surgeons.

When building the master surgery schedule one has to take into consideration the impact on several resources. All these resources share the following properties:

- they are limited in capacity,

- they are expensive, 
- their consumption pattern depends on the master surgery schedule.

In Gasthuisberg's day surgery operating room complex, twelve such resources could be identified. They can be distinguished in five groups: First of all, certain types of surgery require the patient to be lying and transported in a bed (1). Second, there are the human resources that consist of: three skill-specific groups of nurses $(2,3$ and 4$)$, anaesthetists (5) and anaesthetist-supervisors (6). Third, some surgical interventions involve expensive material resources: laporoscopic towers (7), artroscopic towers type 1 (8) and type 2 (9) and lasers type 1 (10) and type 2 (11). Finally, there is the radiology department (12).

\section{Graphical user interface}

In this section the graphical user interface (GUI) is presented. The GUI visualizes the surgery schedule and the resulting bed resource use for a given master surgery schedule. Moreover, it allows the user to modify an existing schedule and view the impact of a change in the schedule on the use of the various resources. Data like the schedule properties, the surgeon properties and the link between the resource utilizations and the block allocations can easily be read in and modified. Figure 2 shows an overview of the GUI with the current surgery schedule for the odd weeks.

The main window is divided into two views. On the left, the master surgery schedule is shown. The columns in the grid represent the time periods from Monday am to Friday $\mathrm{pm}$. The eight rows represent the eight operating rooms X1-X4 and Z1-Z4. Above the grid a legend with the surgical groups is shown. Each surgical group has its own color and style. In this case the style refers to the type of anaesthetic. If the patients are completely anaesthetized during surgery, the surgeon block is colored solidly. Otherwise, when the patients are not fully anaesthetized, the block is arced. The schedule can easily be built from scratch by dragging and dropping the surgeons to the timetable cells.

Each assignment introduces a demand for resources in the system. A subset of these 


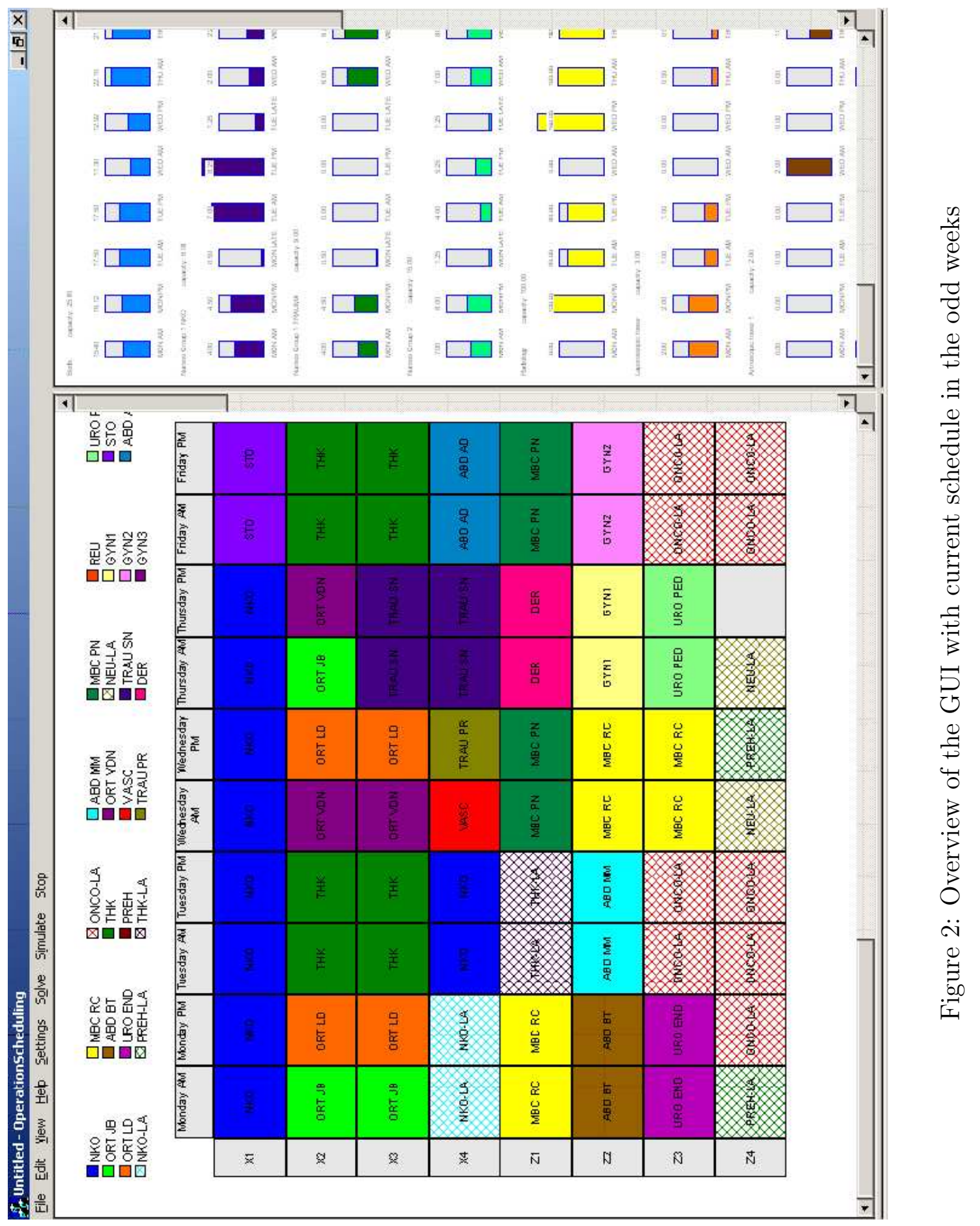


resource utilizations is represented in the right view. Each resource has its own color and time horizon, of which the granularity does not necessarily coincide with that from the surgery cycle time horizon. In our case study, e.g., for the nursing resources on each day an extra time unit is added after the afternoon block. This extra resource unit represents the late shift. Furthermore, for each resource a capacity can be specified that is not necessarily fixed over the total time horizon. In the left view, the scheduler can easily exchange two block assignments by dragging and dropping. In the right view, it will be immediately clear how these changes influence the need for the various resources in the time horizon. In this way the scheduler can quickly detect possible resource conflicts and easily search for workable schedules. Figure 3 provides a more detailed view on the resource consumption patterns.

The second, third and fourth resource are groups of nurses, each having a different speciality (respectively "Group 1 NKO", "Group 1 TRAUMA" and "Group 2"). Each block is colored in proportion to the capacity used. Observe that the need for nurses from "Group 1 NKO" exceeds the indicated capacity on Tuesday and on Friday. This, however, does not necessarily mean that there is a shortage of nurses during these days. The indicated capacities are just leveled targets. When the surgery schedule gives rise to peaks in the demand for nurses, it may be more difficult to schedule the nurses accordingly. In the example shown, nurses have to be shifted from low demand days (Wednesday and Thursday) to peak days (Tuesday and Friday). To obtain efficient schedules, it is very important to have a good integration between the nurse scheduling process and the master surgery scheduling process. A specific model and algorithmic solution procedure to realize this integration is proposed in Beliën and Demeulemeester (2005).

Using dialog boxes, the schedule, surgeon and resource properties could easily be modified. As an example some of the dialog boxes for editing the surgeon properties are presented in Figure 4. The left dialog box shows the surgeon basic properties and a list of the resources that are consumed by the selected surgeon. The user can select one of these resources to edit. The right dialog box then allows the user to indicate how 


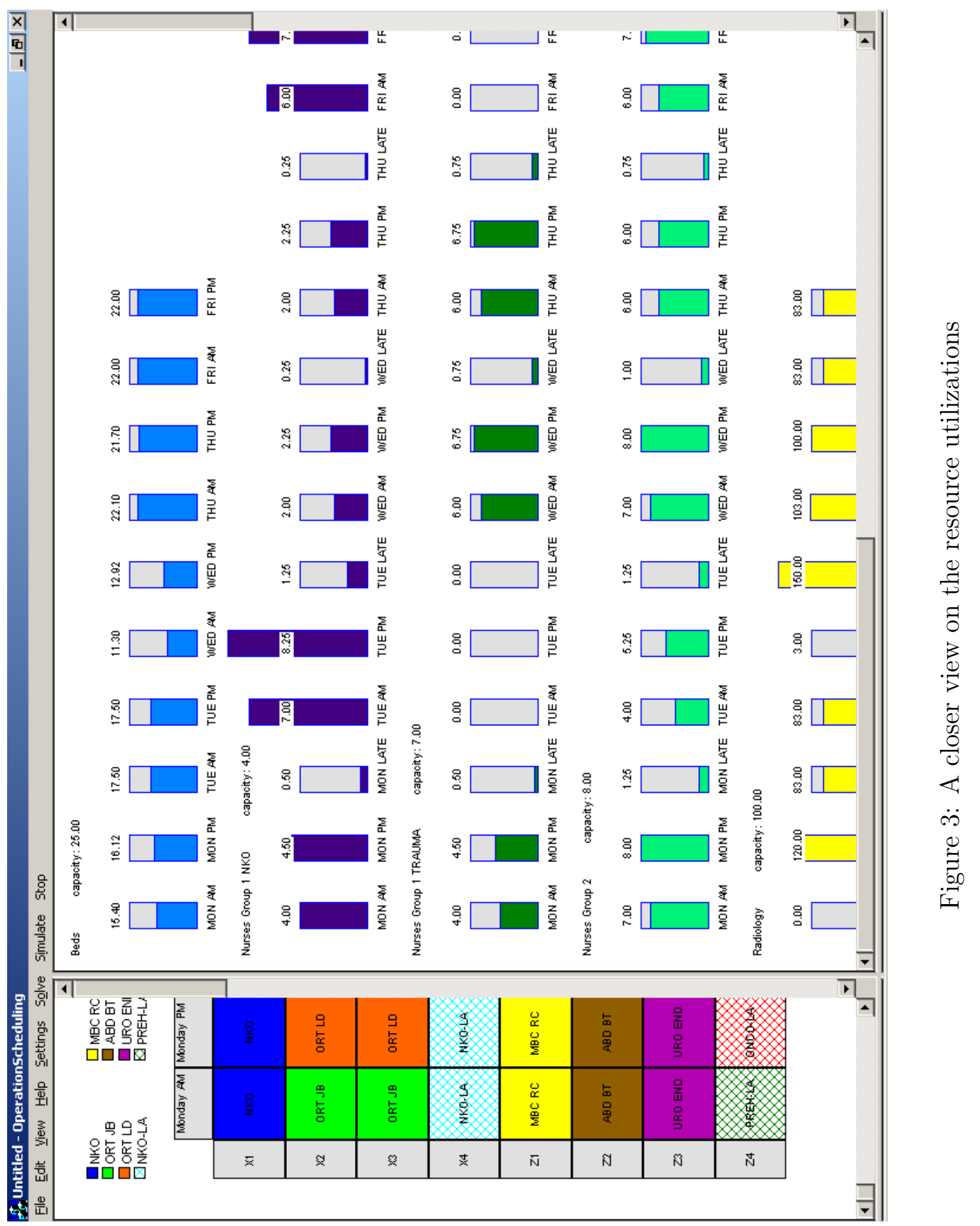


many units and at what moment in time these resources are used by the surgeon (or surgical group). The time index 0 indicates the starting time of the block allocated to the surgeon. In the example shown in Figure 4, two nurses from "Group 1 NKO" are needed to cover the work during surgery time (time index 0) and 1/4 nurse is needed to provide services to operated patients one time period later (pm shift for am surgery or late shift for pm surgery).

The person that is responsible for the operating room schedule of the Gasthuisberg surgical day center evaluated the software during a couple of weeks. His main suggestion for improvement was the ability to have a clear view on all the resources used during each time period given a particular surgery schedule. Accordingly, this feature has been added. Figure 5 contains the same schedule, but this time the resource consumption is presented on a 'per day' view instead of on a 'per resource' view. The user can now easily switch between both views, dependent on the information required. 


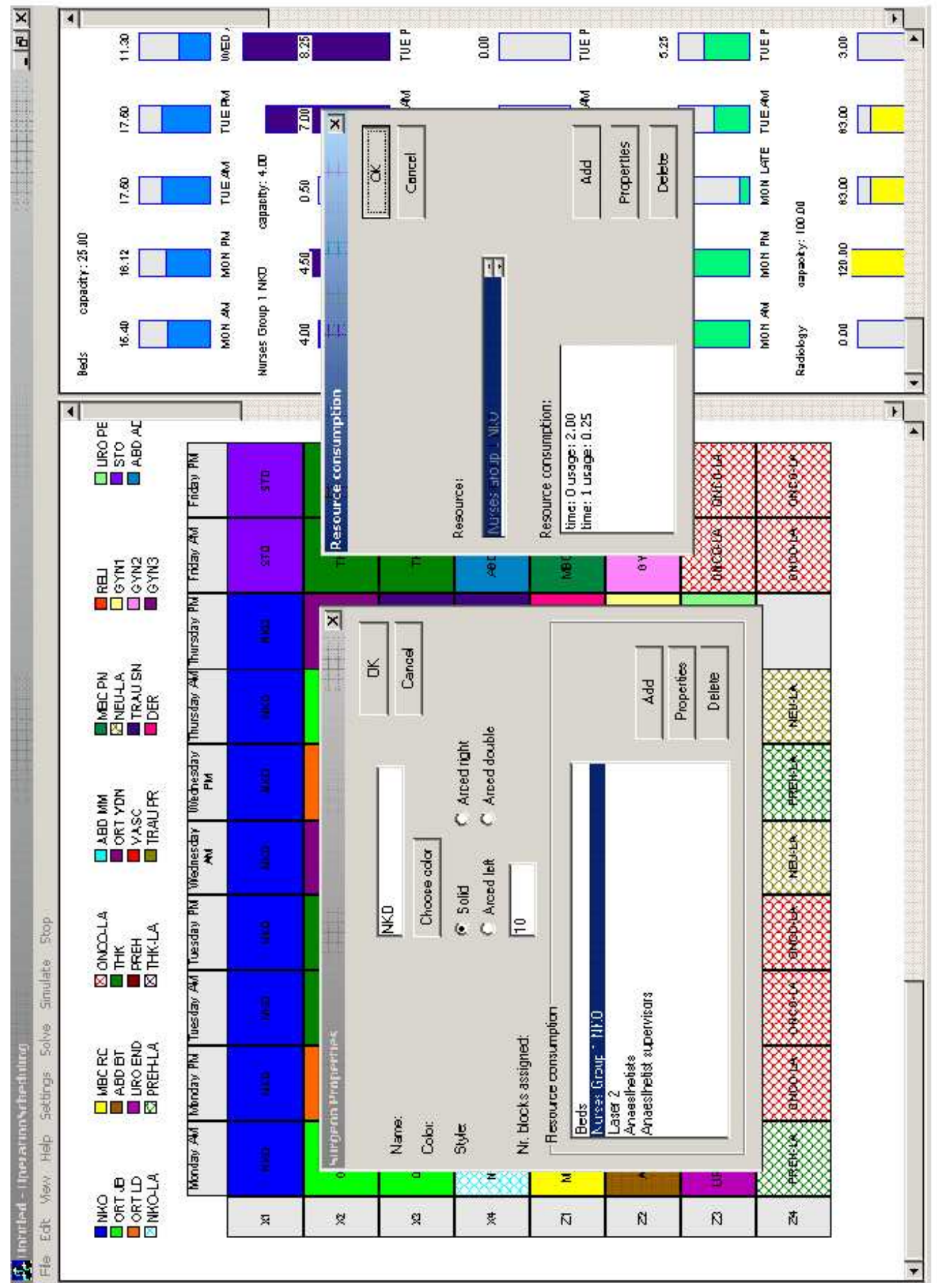

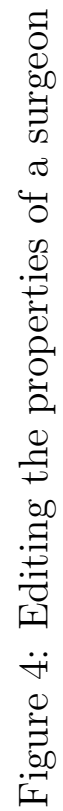




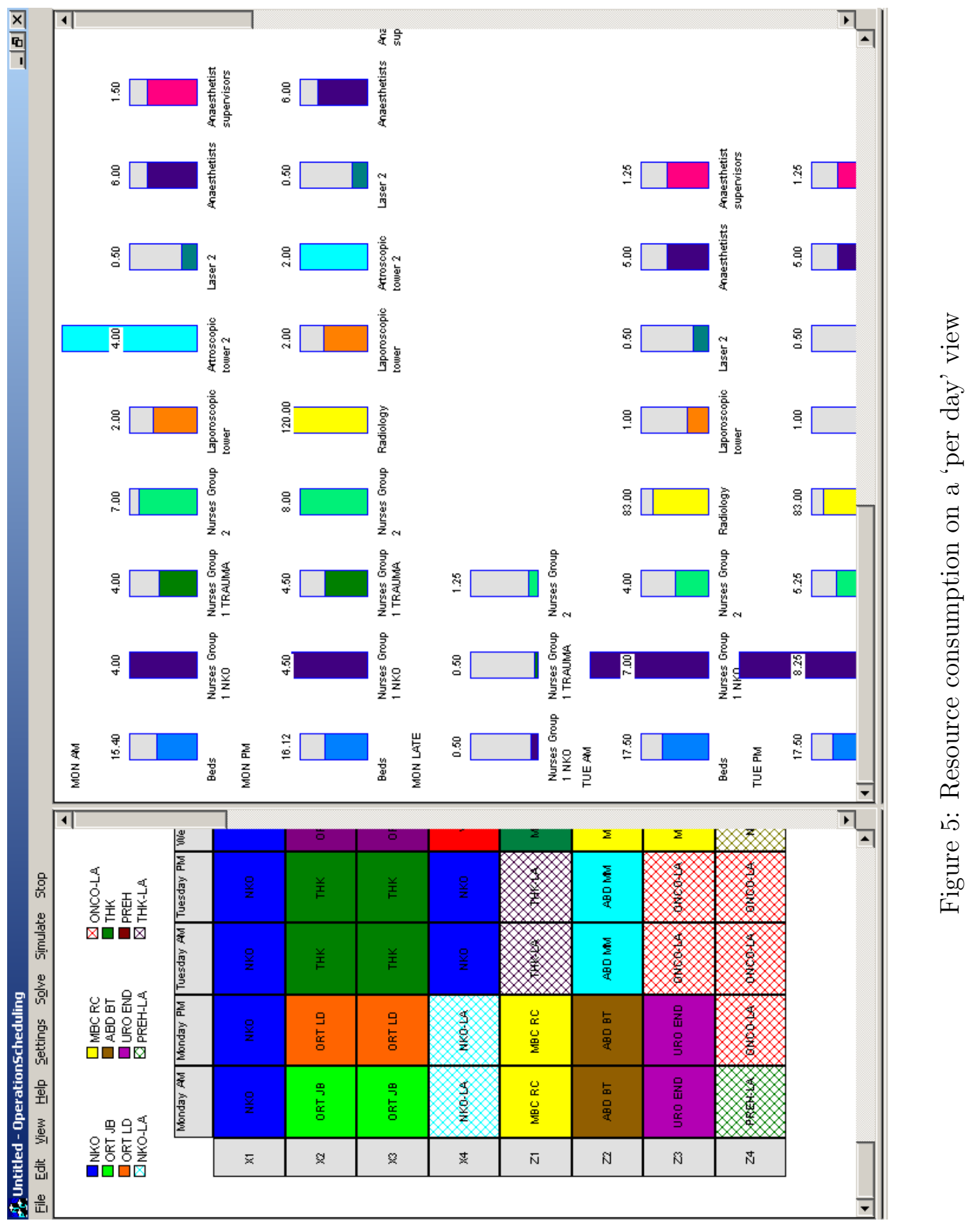




\section{Conclusions and future research}

This paper has presented a visualization system for medical surgery units. Given a particular surgery schedule, the system allows for the visualization of the consumption patterns for a variety of resources. Changes in the schedule are immediately reflected in the periodic resource utilizations. The objective of the system is threefold. First of all, it facilitates the detection of resource conflicts and helps the scheduler to develop workable operating room schedules. Second, the system can greatly assist during the master surgery schedule bargaining process. Third, the system can be of great value for persuading hospital managers to invest in extra resource capacity.

The system is designed for the second stage in building surgery schedules which involves the development of a master surgery schedule. It does not provide an online visualization of available and occupied resources during the daily working of a surgery hospital. It is neither a simulation package for analyzing the existing system and a limited number of alternative scenarios. Instead, our system is deterministic and simple. The extremely intuitive graphical user interface makes it very easy to develop high-quality master surgery schedules. To this aim, schedulers can easily switch block allocations and immediately see the consequences on the consumption of various resources on a cyclic time axis.

The model has been extensively tested and evaluated in the surgical day center of a major Belgian university hospital. The system is considered to be very promising for facilitating the development of the master surgery schedule and for improving the efficiency of resource utilization.

In the current version of our software, all resources are of the renewable type and are treated similarly. Resources could, however, further be classified into certain resource categories having similar characteristics. Think, for instance, of resources that can be shared simultaneously by one or more surgeons whilst other resources cannot. Another example are resources with deterministic utilization, that is the load can be predicted accurately, opposed to resources of which the utilization is subject to high uncertainty. 
The use of equipment is typically deterministic, whereas the bed occupancy is in many cases difficult to predict, due to the uncertainty in the patient's length of stay. It would be interesting to specify several resource categories and enhance the visualization software with dedicated features per resource category.

\section{Acknowledgements}

We are grateful to Pierre Luysmans and Joëlle Baré of the surgical day center of the university hospital Gasthuisberg, for providing the case study data. Special thanks go to Pierre Luysmans for suggesting numerous improvements concerning the functionality of the software. We are indebted to Prof. Dr. Guy Bogaert for his enthusiasm and interest in this project. We acknowledge the support given to this project by the Fonds voor Wetenschappelijk Onderzoek (FWO) - Vlaanderen, Belgium under contract number G.0463.04.

\section{References}

Beliën, J. and Demeulemeester, E. (2005). Integrating nurse and surgery scheduling, Research Report OR 0526, Katholieke Universiteit Leuven, Department of Applied Economics.

Beliën, J. and Demeulemeester, E. (2006). Building cyclic master surgery schedules with leveled resulting bed occupancy. To appear in European Journal of Operational Research.

Blake, J. T. and Carter, M. W. (2002). A goal programming approach to strategic resource allocation in acute care hospitals, European Journal of Operational Research 140: 541-561.

Blake, J. T. and Carter, M. W. (2003). Physician and hospital funding options in a public system with decreasing resources, Socio-Economic Planning Sciences 37: 45-68.

Blake, J. T., Dexter, F. and Donald, J. (2002). Operating room manager's use of integer programming for assigning block time to surgical groups: A case study, Anesthesia and Analgesia 94: 143-148.

Blake, J. T. and Donald, J. (2002). Mount Sinai hospital uses integer programming to allocate operating room time, Interfaces 32: 63-73.

Carter, J. (2000). Timing is everything in the OR, Health Management Technology 21: 80-81.

Clerkin, D., Fos, P. J. and Petry, F. E. (1995). A decision-support system for hospital bed assignment, Hospital and Health Services Administration 40: 386-400. 
Demeulemeester, E. and Herroelen, W. S. (2002). Project scheduling - A research handbook, Kluwer Academic Publishers, Boston.

Dexter, F., Macario, A. and O’Neill, L. (2000). Scheduling surgical cases into overflow block time - Computer simulation of the effects of scheduling strategies on operating room labor costs, Anesthesia and Analgesia 90: 980-988.

Dexter, F., Macario, A. and Traub, R. D. (1999). Which algorithm for scheduling add-on elective cases maximizes operating room utilization?, Anesthesiology 91: 1491-1500.

Dexter, F. and Traub, R. D. (2000). Determining staffing requirements for a second shift of anesthetists by graphical analysis of data from operating room information systems, Anesthesia and Analgesia 68: $31-36$.

Dexter, F. and Traub, R. D. (2002). How to schedule elective surgical cases into specific operating rooms to maximize the efficiency of use of operating room time, Anesthesia and Analgesia 94: 933-942.

Dexter, F., Traub, R. D. and Lebowitz, P. (2001). Scheduling a delay between different surgeons' cases in the same operating room on the same day using upper prediction bounds for case durations, Anesthesia and Analgesia 92: 943-946.

Dumas, M. (1984). Simulation modeling for hospital bed planning, Simulation 8: 69-78.

Dumas, M. (1985). Hospital bed utilization: An implemented simulation approach to adjusting and maintaining levels, Health Services Research 20: 43-61.

Duraiswamy, N., Welton, R. and Reisman, A. (1981). Using computer simulation to predict ICU staffing needs, Journal of Nursing Administration 11: 39-44.

Gorunescu, F., McClean, S. I. and Millard, P. H. (2002). A queueing model for bed-occupancy management and planning of hospitals, Journal of the Operational Research Society 53: 19-24.

Griffiths, J. D., Price-Lloyd, N., Smithies, M. and Williams, J. E. (2005). Modelling the requirement for supplementary nurses in an intensive care unit, Journal of the Operational Research Society 56: $126-133$.

Guinet, A. and Chaabane, S. (2003). Operating theatre planning, International Journal of Production Economics 85: 69-81.

Hamilton, D. M. and Breslawski, S. (1994). Operating room scheduling: Factors to consider, Association of Operating Room Nurses Journal 59: 665-680. 
Hans, E. W., Wullink, G., van Houdenhoven, M. and Kazemier, G. (2005). Robust surgery loading, Technical Report Beta-wp141, dep. Operational Methods for Production and Logistics, University of Twente.

Harris, R. A. (1985). Hospital bed requirements planning, European Journal of Operational Research 25: 121-136.

Hughes, W. L. and Soliman, S. Y. (1985). Short-term case mix management with linear programming, Hospital and Health Services Administration 30: 52-60.

Jun, J. B., Jacobson, S. H. and Swisher, J. R. (1999). Applications of discrete event simulation in health care clinics: A survey, Journal of the Operational Research Society 50: 109-123.

Klein, R. W., Dittus, R. S., Roberts, S. D. and Wilson, J. R. (1993). Simulation modeling and healthcare decision making, Medical Decision Making 13: 347-354.

Lapierre, S. D., Batson, C. and McCaskey, S. (1999). Improving on-time performance in health care organizations: A case study, Health Care Management Science 2: 27-34.

Litvak, E. and Long, M. C. (2000). Cost and quality under managed care: Irreconcilable differences?, The American Journal of Managed Care 6: 305-312.

Marcon, E., Kharraja, S. and Simonnet, G. (2003). The operating theatre planning by the follow-up of the risk of no realization, International Journal of Production Economics 85: 83-90.

McManus, M. L., Long, M. C., Cooper, A. and Litvak, E. (2004). Queuing theory accurately models the need for critical care resources, Anesthesiology 100: 1271-1276.

Ozkarahan, I. (1995). Allocation of surgical procedures to operating rooms, Journal of Medical Systems 19(4): $333-352$.

Ozkarahan, I. (2000). Allocation of surgeries to operating rooms using goal programming, Journal of Medical Systems 24(6): 339-378.

Rifai, A. K. and Pecenka, J. O. (1989). An application of goal programming in healthcare planning, International Journal of Production Management 10: 28-37.

Robbins, W. A. and Tuntiwongbiboon, N. (1989). Linear programming is a useful tool in case-mix management, Healthcare Financial Management 43: 114-116.

Santibanez, P., Begen, M. and Atkins, D. (2005). Managing surgical waitlists for a British Columbia health authority, Research report, Centre for Operations Excellence, Sauder School of Business, University of British Columbia, Canada. 
Standridge, C. R. (1999). A tutorial on simulation in health care: applications issues, WSC '99: Proceedings of the 31st conference on Winter simulation, ACM Press, New York, NY, USA, pp. 4955.

Strum, D. P., Vargas, L. G. and May, J. H. (1997). Resource coordination systems for surgical services using distributed communications, Journal of the American Medical Informatics Association 4: $125-135$.

Swisher, J. R., Jacobson, S. H., Jun, J. B. and Balci, O. (2001). Modeling and analyzing a physician clinic environment using discrete-event (visual) simulation, Computers and Operations Research 28: $105-125$.

Vissers, J. M. H., Bertrand, J. and de Vries, G. (2001). A framework for production control in healthcare organisations, Production Planning and Control 12(6): 591-604.

Weiss, E. N. (1990). Models for determining estimated start times and case orderings in hospital operating rooms, IIE Transactions 22: 143-150.

Wright, M. B. (1987). The application of a surgical bed simulation model, European Journal of Operational Research 32: 26-32. 